\title{
Investigation of Co-Doped $\mathrm{BaTiO}_{3}$ by Atomic-Resolution EELS
}

\author{
Desai. Zhang ${ }^{1}$, T. Aoki ${ }^{2}$, P. Ponath ${ }^{3}$, A. B. Posadas ${ }^{3}$, A. A. Demkov ${ }^{3}$ and D. J. Smith ${ }^{4}$ \\ 1. School of Engineering for Matter, Transport and Energy, Arizona State University, Tempe, AZ 85287 \\ 2. LeRoy Eyring Center for Solid State Science, Arizona State University, Tempe, AZ 85287 \\ 3. University of Texas at Austin, Department of Physics, Austin, Texas 78712 \\ 4. Department of Physics, Arizona State University, Tempe, AZ, 85287
}

$\mathrm{BaTiO}_{3}$ (BTO) is a prototypical ferroelectric perovskite that is an attractive candidate for applications such as negative-capacitance field effect devices [1]. Using $\mathrm{SrTiO}_{3}$ (STO) grown by molecular beam epitaxy as a buffer layer, high quality BTO films can be grown on Si (001) by atomic layer deposition (ALD) [2]. In addition, when BTO is doped with cobalt (Co), its room-temperature dielectric permittivity increases with doping [3]. In this work, Co-doped BTO (BCTO) films were grown on STO buffer layers on Ge (001) substrates. For microscopy characterization, cross-section TEM samples were prepared using mechanical polishing, dimpling and argon-ion-milling.

The film growth quality was checked by high-resolution TEM images, such as Fig. 1, recorded using a JEM-4000EX operated at $400 \mathrm{keV}$ with a structural resolution of $\sim 1.7 \AA$. In addition to the high quality epitaxial layer, a flat STO top surface and an abrupt transition from STO to BCTO are visible. To determine the Co dopant distribution, the sample was examined using Nion UltraSTEM 100 operated at $60 \mathrm{keV}$ to avoid possible beam damage. This microscope is also equipped with a special monochromator that offers energy resolution of $<20 \mathrm{meV}$ for EELS investigations [4]. As shown in Fig. 2, EELS compositional mapping of Co establishes a uniform elemental distribution. Because Co substitutes for Ti in the BTO lattice, the effect of Co doping on the Ti valence state is an important factor. Knowing that $\mathrm{Ti}^{3+}$ and $\mathrm{Ti}^{4+}$ cause different $\mathrm{Ti}-\mathrm{L}_{2,3}$ EELS edge fine structure [5], the Ti oxidation state of BCTO was studied and the results are shown in Fig. 3. Figure 3 (a) shows EELS line scans taken from an area with thickness of $\sim 30 \mathrm{~nm}$, which suggests that $\mathrm{Ti}^{3+}$ is present in BCTO but not in STO. However, EELS line scans taken on thicker areas show the existence of only $\mathrm{Ti}^{4+}$ in the film, as visible in Fig. 3 (b). Moreover, it was found that bypassing the monochromator could result in different morphology of the Ti- $\mathrm{L}_{2,3}$ edge. Two distinct peaks sometimes appeared to be visible when using the monochromator unlike spectra recorded with the monochromator bypassed. This difference is not yet understood and is under further investigation.

In summary, this work has established that the Co dopant is distributed uniformly in the BCTO layer, but raises concerns that sample thickness may cause conflicting results that need to be understood when analyzing the Ti oxidation state in BCTO films. Further observations are in progress [6].

[1] S. Salahuddin and S. Datta, Nano Lett. 8, 405-410 (2008).

[2] M. D. McDaniel, et al, J. Appl. Phys. 115, 224108 (2014).

[3] P. Barik, et al, J. Amer. Ceram. Soc. 94, 2119-2125 (2011).

[4] O. L. Krivanek, et al., Microscopy 62, 3-21 (2013).

[5] Y. Shao, et al, Ultramicroscopy 110, 1014-1019 (2010). 
[6] This work was supported by AFOSR Grant FA9550-12-10494. We gratefully acknowledge the use of facilities within the John M. Cowley Center for High Resolution Electron Microscopy at Arizona State University.
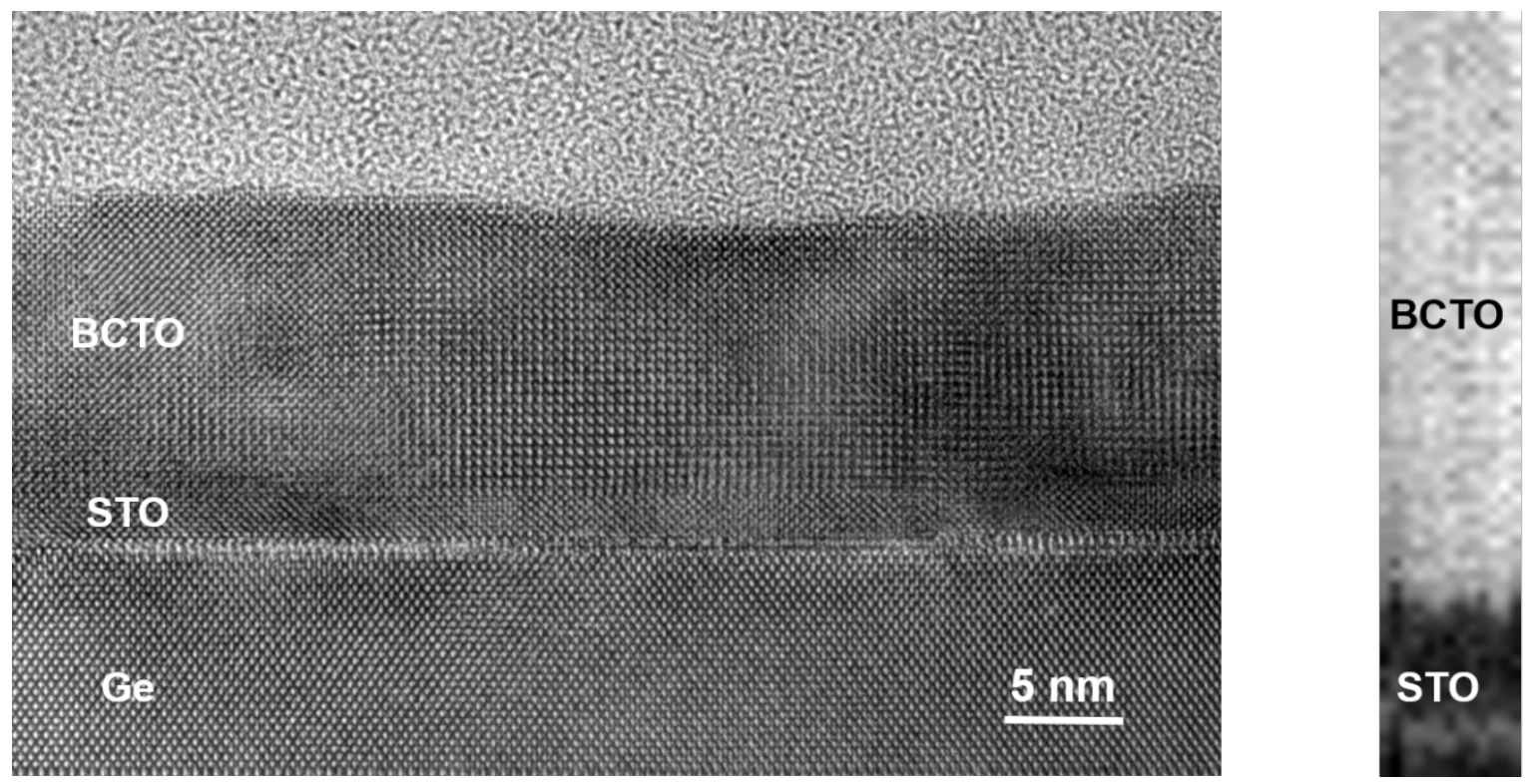

Figure 1. Cross-section high-resolution TEM image of BCTO/STO/Ge(001) sample.

Figure 2. EELS composition map of Co, showing uniform Co distribution.
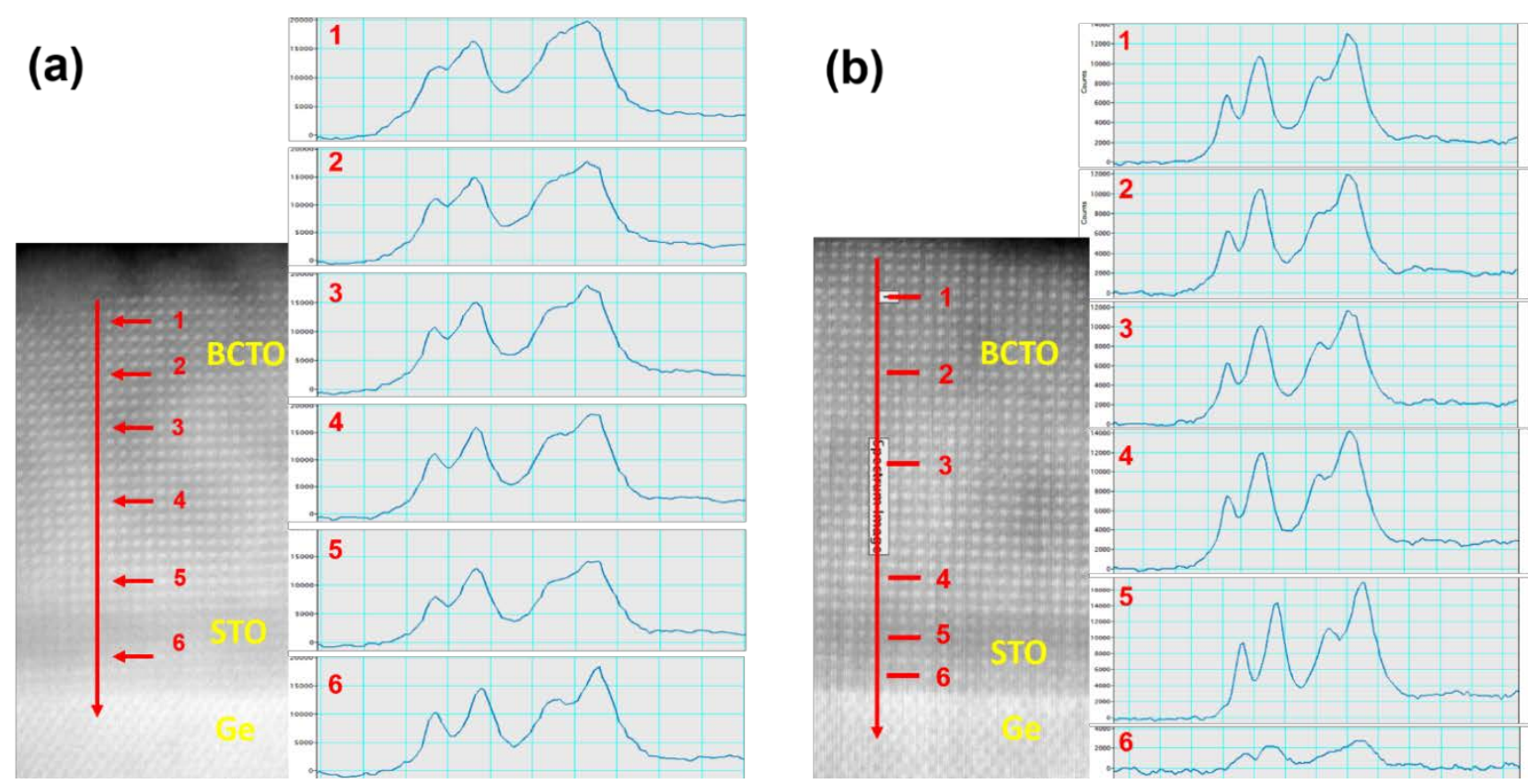

Figure 3. EELS line scans from: (a) thin; and (b) thick, areas of BCTO/STO/Ge(001) sample with monochromator slit inserted. 